Methods Patients presenting with recurrent genital herpes were included in the study. After a complete clinical and laboratory evaluation, the patients were treated with acyclovir $1 \mathrm{gm}$ twice daily orally for 3 days and followed up on days 3,5 and 7 to determine the response to treatment and adverse effects.

Results There were 23 patients $(21$ males and 2 females, between 18-55 years of age), of which 22 complete the study. Nine $(41 \%)$ of them had complete healing of the ulcer on day 3 , whereas $17(77 \%)$ and 20 (91\%) had it by day 5 and 7 respectively. Mean percentage healing of ulcer was $77.95 \pm 26.03$, $90.00 \pm 16.20$ and $95 \pm 7.07$ on day 3,5 , and 7 respectively. Visual analogue score (VAS) showed complete improvement in VAS in 9 (41\%) patients on day 3, 21 (95.5\%) on day 5 and 22 $(100 \%)$ on day 7 . The mean time of complete improvement in VAS was $4.27 \pm 1.16$ days. Mean of percentage improvement in VAS was $80.45 \pm 25.30$ on day 3 and $100 \pm 0.00$ on day 7 Mean healing time of the lesions was $4.67 \pm 1.87$ days (range 3-10 days). There were no significant adverse effects of the therapy.

Conclusions The study demonstrated that oral acyclovir $1 \mathrm{gm}$ twice daily is effective and safe for the treatment of recurrent genital herpes. There was rapid healing of lesions, which reduces morbidity, psychological distress and risk of transmission of infections to sexual partner. Further studies are however needed to confirm our results.

\section{P10.19 CAN HSV-2 SEROPOSITIVITY BE USED AS A BIOLOGICAL MARKER OF SEXUAL BEHAVIOUR? FINDINGS FROM A SEROPREVALENCE SURVEY IN ENGLAND} ${ }^{1,2} \mathrm{SC}$ Woodhall*,${ }^{1} \mathrm{G}$ Murphy, ${ }^{3} \mathrm{R}$ Craig, ${ }^{2} \mathrm{JS}$ Mindell, ${ }^{1} \mathrm{~K}$ Soldan, ${ }^{2} \mathrm{AM}$ Johnson ${ }^{\#}$,
${ }^{1} \mathrm{~A}$ Nardone ${ }^{\#} .{ }^{1}$ Public Health UK; ${ }^{2} \mathrm{UCL} ;{ }^{3}$ NatCen Social Research; ${ }^{\#}$ Joint Last Authors

10.1136/sextrans-2015-052270.447

Introduction Reliable measures of sexual behaviour aid understanding of differences in sexually transmitted infections between groups or over time, but are often difficult or infeasible to collect. Antibody to herpes simplex virus type 2 (HSV-2) has been proposed as a marker of sexual behaviour. We investigated the seroepidemiology of HSV-2 in England to explore the use of HSV-2 as such a marker.

Methods Anonymised sera from 16-44 year-old participants in the Health Survey for England (HSE) in 2010 and 2012, a series of nationally-representative household surveys, were tested for anti-HSV-2 antibodies using the HerpeSelect2 IgG ELISA. Factors associated with seropositivity were investigated using logistic regression

Results HSV-2 seropositivity increased with age up to $15 \%$ (95\% CI: $11 \%-19 \%)$ in women and 9\% (6\%-14\%) in men aged 40-44 years. Seropositivity was higher in those with more sexual partners over the lifetime in women $(1.4 \%$ in those with 0 vs. $14 \%$ in those with $\geq 10$ lifetime sexual partners, $\mathrm{p}=0.018$ ) but not in men $(5 \%$ vs. $7 \%, \mathrm{p}=0.683)$. There was no difference by number of partners in the last year or by recent condom use. Women of black or black British ethnicity had around 3-times higher odds of being seropositive than those of white ethnicity ( $24 \%$ vs. $10 \%$, odds ratio [OR]:2.96, 95\% CI: 1.16-7.54; p = $0.023)$. No difference by ethnicity was noted among men.

Conclusion Our findings suggest antibodies to HSV-2 may be a useful marker for number of lifetime sexual partners in women but not in men. There was less evidence to support the use of HSV-2 as a marker for recent sexual risk behaviour. These data can be used to power future analyses using HSV-2 as a marker of sexual behaviour in women. Although detailed analyses by ethnicity were limited by sample size, the differential risk of seropositivity by ethnicity is of interest and warrants further investigation.

Disclosure of interest statement The study was funded by the Health Protection Agency (now part of Public Health England). No pharmaceutical or diagnostic company grants were received in the development of this study.

\section{P10.20 DIGITAL DROPLET PCR (DDPCR) QUANTIFICATION OF HUMAN T-LYMPHOTROPIC VIRUS TYPE-1 CLADE C IN PERIPHERAL BLOOD AND LUNG INFILTRATES OF INDIGENOUS AUSTRALIAN BRONCHIECTASIS PATIENTS}

${ }^{1}$ D Yurick*, ${ }^{2}$ L Einsiedel, ${ }^{2} \mathrm{H}$ Pham, ${ }^{1} \mathrm{G}$ Khoury," ${ }^{1} \mathrm{DFJ}$ Purcell. 'Department of Microbiology, at the Peter Doherty Institute for Infection and Immunity, University of Melbourne, Parkville, VIC; ${ }^{2}$ Northern Territory Rural Clinical School/Flinders University, SA

\subsection{6/sextrans-2015-052270.448}

Background Human T-cell Lymphotropic Virus type-1 (HTLV-1) infects an estimated 20 million people worldwide causing a fatal T- cell leukaemia and inflammatory conditions, such as HTLV-1associated myelopathy, resulting from a dysregulated inflammatory response to the virus. HTLV-1 is highly endemic to remote indigenous communities in Central Australia where infection contributes to racial disparities in morbidity and mortality. HTLV-1 related complications are associated with elevated numbers of T-cells in which the HTLV-1 provirus integrates (HTLV-1 proviral load, PVL). Digital-droplet PCR (ddPCR) is capable of absolute quantification of PVL, offering low variability between assays with few cell numbers. We measured HTLV-1 PVLs from buffy coat cells (BCCs) of infected individuals, and bronchoalveolar lavage (BALs) infiltrates from a bronchiectasis patient to determine the range of HTLV-1 copies/cell relative to illness. Methods All samples were from Alice Springs Hospital HTLV-1 cohort (8 BCCs, 1 BAL) and obtained following ethics approval and patient consent in first language. Genomic DNA was extracted from BCCs for ddPCR PVL quantification. HTLV-1 gag/tax primers and FAM/MGB probes were optimised using temperature gradient amplification. Samples were tested in duplicate relative to an internal reference gene standard, RPP30, or negative threshold controls that used VIC/MGB probe. QuantaSoft software was used for data analysis.

Results Of the 8 samples from BCCs, the PVL fell between 320 - 8,040 proviral copies per $10^{6}$ cells. The BAL sample PVL was 1,110 DNA copies per $10^{6}$ cells. The sensitivity (or limit of detection) of our ddPCR assay is 2 copies of proviral DNA per $10^{4}$ cells.

Conclusion The ddPCR assay demonstrates extremely high sensitivity, low assay-variability and the capability to reliably quantify HTLV-1 PVL. This technique offers logistic advantages in studying relationships between PVL in HTLV-1 patient's BCCs and BAL.

Disclosure of interest statement No Conflicts of interest.

ISSTDR and IUSTI recognise the considerable contribution that industry partners make to professional and research activities. We also recognise the need for transparency of disclosure of potential conflicts of interest by acknowledging these relationships in publications and presentations.

No commercial contributions have been received for this research project. 